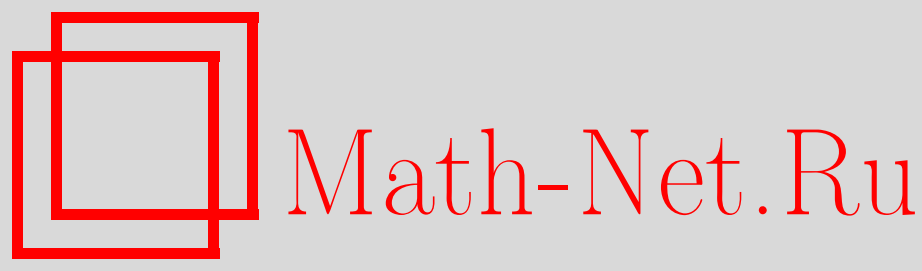

А. В. Перескоков, Квазиклассическая асимптотика спектра оператора типа Хартри вблизи верхних границ спектральных кластеров, ТМФ, 2014, том 178, номер 1, 88106

DOI: https://doi.org/10.4213/tmf8561

Использование Общероссийского математического портала Math-Net.Ru подразумевает, что вы прочитали и согласны с пользовательским соглашением http://www.mathnet.ru/rus/agreement

Параметры загрузки:

IP: 52.87 .193 .239

26 апреля 2023 г., 09:26:34

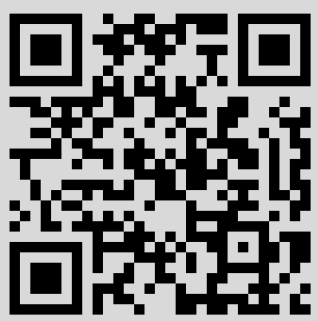




\title{
ФИЗИКА
}

Том 178, № 1

январь, 2014

(C) 2014 г.

А. В. Перескоков ${ }^{* \dagger}$

\section{КВАЗИКЛАССИЧЕСКАЯ АСИМПТОТИКА СПЕКТРА ОПЕРАТОРА ТИПА ХАРТРИ ВБЛИЗИ ВЕРХНИХ ГРАНИЦ СПЕКТРАЛЬНЫХ КЛАСТЕРОВ}

\begin{abstract}
Рассматривается задача на собственные значения для возмущенного двумерного осциллятора в случае резонанса частот. Возбуждающий потенциал задается интегральной нелинейностью типа Хартри с гладким потенциалом самодействия. Найдены асимптотические собственные значения и асимптотические собственные функции вблизи верхних границ спектральных кластеров, которые образуются вокруг уровней энергии невозмущенного оператора. Для их вычисления использованы асимптотические формулы квантовых средних.
\end{abstract}

Ключевые слова: самосогласованное поле, метод квантового усреднения, когерентное преобразование, ВКБ-приближение, спектральный кластер, квантовое среднее.

DOI: $10.4231 / \operatorname{tmf} 8561$

\section{1. ВВЕДЕНИЕ}

Рассмотрим задачу на собственные значения для нелинейного оператора типа Хартри в пространстве $L^{2}\left(\mathbb{R}^{2}\right)$ :

$$
\left(\mathbf{H}_{0}+\varepsilon \int_{\mathbb{R}^{2}} W\left(\left|q-q^{\prime}\right|^{2}\right)\left|\psi\left(q^{\prime}\right)\right|^{2} d q^{\prime}\right) \psi=\lambda \psi, \quad\|\psi\|_{L^{2}\left(\mathbb{R}^{2}\right)}=1,
$$

где

$$
\mathbf{H}_{0}=-\frac{\hbar^{2}}{2}\left(\frac{\partial^{2}}{\partial q_{1}^{2}}+\frac{\partial^{2}}{\partial q_{2}^{2}}\right)+\frac{q_{1}^{2}+q_{2}^{2}}{2}
$$

- двумерный осциллятор, $W(x)=w_{0}+w_{1} x+w_{2} x^{2}-$ произвольный многочлен второй степени с вещественными коэффициентами, $\hbar>0, \varepsilon>0$ - малые параметры, причем $\varepsilon \ll \hbar$. Для определенности рассмотрим случай $\varepsilon=\hbar^{2}$ и $w_{2}>0$.

*Федеральное государственное бюджетное образовательное учреждение высшего профессионального образования Национальный исследовательский университет МЭИ, Москва, Россия. E-mail: pereskokov62@mail.ru

${ }^{\dagger}$ Национальный исследовательский университет Высшая школа экономики Московский институт электроники и математики "МИЭМ НИУ ВШЭ”, Москва, Россия 
Уравнение самосогласованного поля во внешнем поле с гладким потенциалом самодействия $W$ играет фундаментальную роль в квантовой теории и нелинейной оптике, в частности в теории конденсата Бозе-Эйнштейна [1] и при описании коллективных возбуждений в молекулярных цепочках и в молекулах ДНК [2].

Асимптотическим решениям уравнений типа Хартри, локализованным вблизи маломерных инвариантных подмногообразий в фазовом пространстве, посвящено большое число работ (см., например, [3]-[12]). Особенностью задачи (1.1) является то, что она относится к классу резонансных: обе частоты двумерного осциллятора $\mathbf{H}_{0}$ равны единице. Но в этом случае лучевой метод [13] и общая теория комплексного ростка Маслова [3], которые позволяют строить асимптотические при $\hbar \rightarrow 0$ решения, локализованные вблизи точек, траекторий, торов и иных маломерных подмногообразий, неприменимы [14].

Метод построения квазиклассических асимптотик для уравнений с частотными резонансами был разработан в серии работ М. В. Карасева [15]-[17]. Он основан на алгебраическом усреднении возмущения, последующем переходе на алгебру симметрий и когерентном преобразовании от исходного представления этой алгебры к ее неприводимому представлению в пространстве функций над лагранжевым подмногообразием в симплектическом листе.

Особый интерес представляют решения уравнений типа (1.1), отвечающие границам спектральных кластеров вблизи собственных значений невозмущенного уравнения (при $\varepsilon=0)$, где упомянутые лагранжевы подмногообразия почти схлопываются, и интегральное представление решения над ними становится невозможным. В работах [18], [19] на примере спектральной задачи для двумерного возмущенного осциллятора был предложен метод нахождения серий асимптотических собственных значений вблизи границ спектральных кластеров. Он основан на новом интегральном представлении (см. формулу (3.8) настоящей работы). В работах [20], [21] этот метод был использован при изучении асимптотики спектра атома водорода в магнитном поле.

В настоящей работе указанный метод применен для нахождения асимптотических собственных значений оператора типа Хартри вблизи верхних границ спектральных кластеров (см. теорему 1). В силу нелинейности оператора собственным значениям соответствуют однопараметрические семейства асимптотических собственных функций. Помимо нелинейности, имеется еще целый ряд свойств, отличающих задачу (1.1) от рассмотренных ранее. Например, после усреднения и когерентного преобразования в случае уравнения (1.1) получается уравнение, которое имеет иррегулярные особые точки (см. уравнение (3.3) настоящей работы). Оно не является уравнением класса Фукса [22], в отличие от работ [18]-[21].

Задача, подобная (1.1), но без резонансов рассматривалась ранее в работе [5], где для оператора типа Хартри была построена асимптотика серии собственных значений. Эта серия соответствует радиально несимметричным собственным функциям с большим орбитальным числом, произвольным магнитным числом и с небольшим радиальным числом. Отметим также работы [10], [11], где для небольших квантовых чисел была найдена серия асимптотических собственных значений для оператора типа Хартри. Она отвечает точке покоя системы Гамильтона-Эренфеста, ассоциированной с оператором Хартри. 


\section{2. АЛГЕБРАИЧЕСКОЕ УСРЕДНЕНИЕ И КОГЕРЕНТНОЕ ПРЕОБРАЗОВАНИЕ}

Заметим, что оператор в левой части уравнения (1.1) можно записать в виде

$$
\mathbf{H}_{0}+\hbar^{2} V\left(q_{1}, q_{2}\right),
$$

где $V\left(q_{1}, q_{2}\right)$ - многочлен четвертой степени от $q_{1}, q_{2}$, коэффициенты которого интегрально зависят от $|\psi|^{2}$. Применим к уравнению (1.1) квантовую версию метода усреднения [14], [23]. Исходная идея метода состоит в том, чтобы найти такой обратимый оператор $\mathbf{U}$ и такой оператор $\mathbf{V}_{0}$, чтобы были выполнены равенства

$$
\mathbf{U}^{-1}\left(\mathbf{H}_{0}+\hbar^{2} V\right) \mathbf{U}=\mathbf{H}_{0}+\hbar^{2} \underline{\mathbf{V}_{0}}+O\left(\hbar^{4}\right), \quad\left[\underline{\mathbf{V}_{0}}, \mathbf{H}_{0}\right]=0 .
$$

Новый возмущающий оператор $\mathbf{V}_{0}$ коммутирует со старшей частью оператора $\mathbf{H}_{0}$. Поэтому решение спектральной задачи для оператора $\mathbf{H}_{0}+\hbar^{2} V$ сводится к решению спектральной задачи для оператора $\mathbf{V}_{0}$ на собственном подпространстве оператора $\mathbf{H}_{0}$. Отметим, что реализовать эту идею удается лишь при достаточно жестких ограничениях на оператор $\mathbf{H}_{0}$. А именно, должно быть выполнено условие

$$
\exp \left\{\frac{2 \pi i}{\hbar} \mathbf{H}_{0}\right\}=I
$$

где $I$ - единичный оператор.

В случае задачи (1.1) оператор $\mathbf{V}_{0}$ может быть представлен в виде

$$
\underline{\mathbf{V}_{0}}=2 w_{2} f\left(\mathbf{S}_{1}, \mathbf{S}_{2}, \mathbf{S}_{3}\right)+b_{0},
$$

где $f$ - многочлен второй степени, $b_{0}$ - константа, а $\mathbf{S}_{1}, \mathbf{S}_{2}, \mathbf{S}_{3}$ - симметризированные по Вейлю "швингеровские" образующие алгебры вращений [24], удовлетворяющие циклическим коммутационным соотношениям

$$
\left[\mathbf{S}_{1}, \mathbf{S}_{2}\right]=i \hbar \mathbf{S}_{3}, \quad\left[\mathbf{S}_{2}, \mathbf{S}_{3}\right]=i \hbar \mathbf{S}_{1}, \quad\left[\mathbf{S}_{3}, \mathbf{S}_{1}\right]=i \hbar \mathbf{S}_{2} .
$$

На гильбертовом подпространстве $\mathcal{H}_{\ell} \subset L^{2}\left(\mathbb{R}^{2}\right)$ собственных функций оператора $\mathbf{H}_{0}$, отвечающих собственному значению $\hbar(\ell+1)$, где $\ell=0,1,2, \ldots$, получаем спектральную задачу

$$
f\left(\mathbf{S}_{1}, \mathbf{S}_{2}, \mathbf{S}_{3}\right) \varphi=\xi \varphi, \quad\|\varphi\|_{\mathcal{H}_{\ell}}=1 .
$$

Ее собственные значения обозначим через $\xi=\xi_{k, \ell}, k=0,1,2, \ldots$, и упорядочим их по убыванию. Рассмотрим число $\ell$ порядка $\hbar^{-1}$. Тогда спектр исходной задачи (1.1) имеет следующую асимптотику:

$$
\lambda=\hbar(\ell+1)+\hbar^{2}\left(2 w_{2} \xi_{k, \ell}+b_{0}\right)+O\left(\hbar^{4}\right) .
$$

Асимптотика соответствующих собственных функций дается формулой

$$
\psi=\mathbf{U} \varphi_{k, \ell}+O\left(\hbar^{3}\right),
$$

где $\varphi_{k, \ell}$ - собственная функция задачи (2.1), которая отвечает собственному значению $\xi_{k, \ell}$. Оператор $\mathbf{U}$ можно найти в явном виде [14], [19]. 
Положим $a=\ell \hbar / 2$. В случае задачи (1.1) вычисления, аналогичные проведенным в работе [19], приводят к формулам для многочлена

$$
f\left(\mathbf{S}_{1}, \mathbf{S}_{2}, \mathbf{S}_{3}\right)=-\mathbf{S}_{2}^{2}+b_{1} \mathbf{S}_{1}+b_{3} \mathbf{S}_{3}
$$

и констант

$$
\begin{gathered}
b_{0}=w_{0}+4 \sqrt{a(a+\hbar)} w_{1}+\left[28 a(a+\hbar)-2\left(\mathbf{S}_{2}^{2} \varphi, \varphi\right)_{\mathcal{H}_{\ell}}\right] w_{2} \\
b_{1}=4\left(\mathbf{S}_{1} \varphi, \varphi\right)_{\mathcal{H}_{\ell}}, \quad b_{3}=4\left(\mathbf{S}_{3} \varphi, \varphi\right)_{\mathcal{H}_{\ell}}
\end{gathered}
$$

Чтобы решить задачу (2.1), воспользуемся когерентным преобразованием [25]

$$
I_{\ell}(g)=\frac{\ell+1}{2 \pi} \int_{\mathbb{C}} g(\bar{z})|z\rangle \frac{d \bar{z} d z}{\left(1+|z|^{2}\right)^{\ell+2}},
$$

где

$$
|z\rangle=\frac{\left(1+z^{2}\right)^{\ell / 2}}{\sqrt{\pi 2^{\ell} \ell ! \hbar}} H_{\ell}\left(\frac{q_{1}+z q_{2}}{\sqrt{\hbar} \sqrt{1+z^{2}}}\right) e^{-\left(q_{1}^{2}+q_{2}^{2}\right) / 2 \hbar},
$$

a $H_{\ell}(q)$ - полиномы Эрмита. Оно отображает гильбертово пространство $\mathcal{P}_{\ell}$ антиголоморфных многочленов степени не выше $\ell$ на гильбертово пространство $\mathcal{H}_{\ell}$. Скалярное произведение в $\mathcal{P}_{\ell}$ задается следующей формулой [25]:

$$
\left(g_{1}, g_{2}\right)_{\mathcal{P}_{\ell}}=\frac{\ell+1}{2 \pi} \int_{\mathbb{C}} g_{1}(\bar{z}) \overline{g_{2}(\bar{z})} \frac{d \bar{z} d z}{\left(1+|z|^{2}\right)^{\ell+2}}
$$

Будем искать решение задачи $(2.1)$ в виде $\varphi_{k, \ell}=I_{\ell}\left(\Phi_{k, \ell}(\bar{z})\right)$. Поскольку в результате когерентного преобразования (2.7) операторы $\mathbf{S}_{1}, \mathbf{S}_{2}, \mathbf{S}_{3}$ преобразуются в дифференциальные операторы первого порядка [25]

$$
\begin{aligned}
& \stackrel{0}{S_{1}}=\frac{\hbar}{2}\left(\bar{z} \ell+\left(1-\bar{z}^{2}\right) \frac{d}{d \bar{z}}\right), \\
& \stackrel{0}{S}_{2}=\frac{i \hbar}{2}\left(\bar{z} \ell-\left(1+\bar{z}^{2}\right) \frac{d}{d \bar{z}}\right), \\
& \stackrel{0}{S}_{3}=\hbar\left(\frac{\ell}{2}-\bar{z} \frac{d}{d \bar{z}}\right),
\end{aligned}
$$

мы получаем следующее уравнение для $\Phi_{k, \ell}(\bar{z})$ :

$$
f\left(\stackrel{0}{S}_{1}, \stackrel{0}{S}_{2}, \stackrel{0}{S}_{3}\right) \Phi_{k, \ell}(\bar{z})=\xi_{k, \ell} \Phi_{k, \ell}(\bar{z}), \quad k=0,1,2, \ldots
$$

Здесь функция $f$ определена формулой (2.4). Назовем собственными числами уравнения (2.10) такие значения параметра $\xi_{k, \ell}$, при которых это уравнение имеет полиномиальное решение в пространстве $\mathcal{P}_{\ell}$. В силу унитарности когерентного преобразования мы имеем

$$
\left\|\Phi_{k, \ell}\right\|_{\mathcal{P}_{\ell}}=1
$$

Пусть $\bar{z}=x_{0}$ - точка, в малой окрестности которой локализована собственная функция $\Phi_{k, \ell}(\bar{z})$. Из предположения о единственности такой точки вытекает, что $x_{0} \in \mathbb{R}$. Покажем, как связана эта точка со спектром оператора Хартри. 
Рассмотрим сужение функции (2.4) на сферу $\Omega_{\ell}$, заданную уравнением

$$
S_{1}^{2}+S_{2}^{2}+S_{3}^{2}=a(a+\hbar)
$$

которая является симплектическим листом для алгебры вращений. Введем на $\Omega_{\ell}$ кэлерову структуру с помощью комплексной координаты

$$
z=\frac{S_{1}+i S_{2}}{\sqrt{a(a+\hbar)}+S_{3}} .
$$

Пусть $\bar{z}$ - функция, комплексно-сопряженная (2.13). Тогда в силу равенств (2.12) и (2.13) мы имеем [25]

$$
\begin{aligned}
& S_{1}=\sqrt{a(a+\hbar)} \frac{z+\bar{z}}{1+|z|^{2}}, \\
& S_{2}=i \sqrt{a(a+\hbar)} \frac{\bar{z}-z}{1+|z|^{2}}, \\
& S_{3}=\sqrt{a(a+\hbar)} \frac{1-|z|^{2}}{1+|z|^{2}} .
\end{aligned}
$$

Далее перейдем в $f$ от координат $S_{1}, S_{2}, S_{3}$ к новым координатам $z, \bar{z}$. В результате сужение функции (2.4) на сферу $\Omega_{\ell}$ примет вид

$$
f_{\Omega_{\ell}}(z, \bar{z})=a(a+\hbar) \frac{(\bar{z}-z)^{2}}{\left(1+|z|^{2}\right)^{2}}+b_{1} \sqrt{a(a+\hbar)} \frac{z+\bar{z}}{1+|z|^{2}}+b_{3} \sqrt{a(a+\hbar)} \frac{1-|z|^{2}}{1+|z|^{2}} .
$$

Чтобы вычислить значения коэффициентов $b_{1}, b_{3}$, воспользуемся формулами для средних значений дифференциальных операторов на решениях задачи (1.1) вблизи границ спектральных кластеров [18], [19]. Поскольку собственные функции $\varphi_{k, \ell}(z, \bar{z})$ локализованы в малой окрестности точки $z=\bar{z}=x_{0}$, для вычисления квантовых средних операторов $\mathbf{S}_{1}$ и $\mathbf{S}_{3}$ достаточно заменить функции в $(2.14)$ их значениями в точке $z=\bar{z}=x_{0}$. В результате в силу формул (2.6) получаем, что при $\hbar \rightarrow 0$

$$
b_{1} \sim 4 \sqrt{a(a+\hbar)} \frac{2 x_{0}}{1+x_{0}^{2}}, \quad b_{3} \sim 4 \sqrt{a(a+\hbar)} \frac{1-x_{0}^{2}}{1+x_{0}^{2}} .
$$

Приведенные выше формальные рассуждения будут далее строго обоснованы (см. лемму 4).

Подставляя асимптотики (2.16) в формулу (2.15), окончательно получаем

$$
f_{\Omega_{\ell}}(z, \bar{z}) \sim a(a+\hbar)\left[\frac{(\bar{z}-z)^{2}}{\left(1+|z|^{2}\right)^{2}}+\frac{8 x_{0}(z+\bar{z})}{\left(1+x_{0}^{2}\right)\left(1+|z|^{2}\right)}+\frac{4\left(1-x_{0}^{2}\right)\left(1-|z|^{2}\right)}{\left(1+x_{0}^{2}\right)\left(1+|z|^{2}\right)}\right] .
$$

Глобальный максимум этой функции достигается в точке $x_{0}$ и равен

$$
4 a(a+\hbar)=4 a^{2}+O(\hbar) .
$$

Число $4 a^{2}$ определяет верхнюю границу спектрального кластера. Далее мы вычислим поправку к этому числу (см. формулу (6.7)). 
Неоднозначность в выборе точки $x_{0}$, вблизи которой локализовано решение, связана с неединственностью собственной функции в задаче (1.1). Действительно, собственные функции инвариантны относительно сдвига полярного угла. Это свойство сохраняется и после усреднения. Следовательно, в пространстве $\mathcal{P}_{\ell}$ также имеется однопараметрическое семейство собственных функций (многочленов), параметризуемое числом $x_{0} \in \mathbb{R}$. Оператор, преобразующий одну собственную функцию в другую, приведен ниже (см. формулу (5.12)). Отметим, что для многомерных нестационарных уравнений типа Хартри операторы симметрии найдены в работе [12].

\section{3. ИНТЕГРАЛЬНОЕ ПРЕДСТАВЛЕНИЕ ДЛЯ АСИМПТОТИЧЕСКИХ СОБСТВЕННЫХ ФУНКЦИЙ}

Рассмотрим уравнение (2.1), где функция $f$ имеет вид (2.4). С учетом соотношений (2.16) будем искать $b_{1}$ и $b_{3}$ в виде

$$
b_{1}=\frac{8 a x_{0}}{1+x_{0}^{2}}+\hbar b_{1}^{(1)}+O\left(\hbar^{2}\right), \quad b_{3}=4 a \frac{1-x_{0}^{2}}{1+x_{0}^{2}}+\hbar b_{3}^{(1)}+O\left(\hbar^{2}\right), \quad \hbar \rightarrow 0 .
$$

Кроме того, положим

$$
\xi_{k, \ell}=4 a^{2}+\hbar \xi_{k, \ell}^{(1)}+O\left(\hbar^{2}\right), \quad \hbar \rightarrow 0
$$

Числа $b_{1}^{(1)}, b_{3}^{(1)}, \xi_{k, \ell}^{(1)}$ будут определены ниже при вычислении поправок в квантовых средних. Учитывая формулы (2.9), а также отбрасывая в разложениях (3.1), (3.2) слагаемые порядка $O\left(\hbar^{2}\right)$, приходим к уравнению (далее для упрощения обозначений индексы $k, \ell$ у функции $\Phi(\bar{z})$ мы опускаем)

$$
\begin{aligned}
& \hbar^{2} R^{2}(\bar{z}) \frac{d^{2} \Phi}{d \bar{z}^{2}}+\left\{-4 a \hbar\left[\bar{z} R(\bar{z})+\frac{4\left(1-x_{0}^{2}\right) \bar{z}-4 x_{0}\left(1-\bar{z}^{2}\right)}{R\left(x_{0}\right)}\right]+\right. \\
& \left.+2 \hbar^{2}\left[\bar{z} R(\bar{z})-2 b_{3}^{(1)} \bar{z}+b_{1}^{(1)}\left(1-\bar{z}^{2}\right)\right]\right\} \frac{d \Phi}{d \bar{z}}+ \\
& \quad+\left\{4 a^{2}\left[\bar{z}^{2}+\frac{8 x_{0}\left(\bar{z}-x_{0}\right)}{R\left(x_{0}\right)}\right]+2 a \hbar\left[-R(\bar{z})+2\left(b_{3}^{(1)}-\frac{\xi_{k, \ell}^{(1)}}{a}\right)+2 b_{1}^{(1)} \bar{z}\right]\right\} \Phi=0 .
\end{aligned}
$$

Здесь

$$
R(\bar{z})=\bar{z}^{2}+1
$$

Уравнение (3.3) имеет две особые точки $\bar{z}_{1}=i$ и $\bar{z}_{2}=-i$, которые являются корнями уравнения $R(\bar{z})=0$, а также особую точку $\bar{z}_{3}=\infty$. Точки $\bar{z}_{1}, \bar{z}_{2}$ являются иррегулярными, а точка $\bar{z}_{3}$ - регулярной.

Уравнения Фукса с тремя особыми точками порождают хорошо известные системы классических ортогональных полиномов [26]. Для уравнения (3.3) подобной теории не существует. 
Нам потребуются некоторые результаты теории когерентных преобразований [25]. Наряду с пространством $\mathcal{P}_{\ell}$ рассмотрим дуальное ему гильбертово пространство $\widetilde{\mathcal{P}}_{\ell}$, состоящее из мероморфных распределений на $\mathbb{C} \backslash\{0\}$ вида

$$
\tilde{g}(z)=\sum_{n=0}^{\ell} \frac{\tilde{g}_{n}}{z^{n+1}} .
$$

Двойственность между пространствами $\widetilde{\mathcal{P}}_{\ell}$ и $\mathcal{P}_{\ell}$ задается отображением

$$
\mathcal{K}: \widetilde{\mathcal{P}}_{\ell} \rightarrow \mathcal{P}_{\ell}, \quad(\mathcal{K} \tilde{g})(\bar{w})=\frac{1}{2 \pi i} \oint_{\gamma} K(\bar{w}, z) \tilde{g}(z) d z,
$$

где воспроизводящее ядро определяется как

$$
K(\bar{w}, z)=(1+\bar{w} z)^{\ell}
$$

а $\gamma$ - замкнутый контур вокруг точки $z=0$, ориентированный против часовой стрелки. Обратное отображение имеет вид

$$
\left(\mathcal{K}^{-1} g\right)(z)=-\frac{1}{2 \pi i} \oint_{\gamma} \widetilde{L}(\bar{w}, z) g(\bar{w}) d \bar{w},
$$

где мероморфное воспроизводящее ядро задается как

$$
\widetilde{L}(\bar{w}, z)=\sum_{n=0}^{\ell} \frac{n !(\ell-n) !}{\ell !(\bar{w} z)^{n+1}} .
$$

Обозначим через $G(\bar{u}, \bar{w})$ ядро суперпозиции операторов $\mathcal{K}^{-1}, \mathcal{K}$. Из формул $(3.5)$ и (3.6) вытекает, что

$$
G(\bar{u}, \bar{w})=\frac{\bar{u}^{\ell+1}-\bar{w}^{\ell+1}}{\bar{u}^{\ell+1}(\bar{u}-\bar{w})} .
$$

Данное ядро в пространстве $\mathcal{P}_{\ell}$ определяет тождественный оператор, а на множестве $J$ антиголоморфных в окрестности нуля функций является проектором на пространство $\mathcal{P}_{\ell}$.

Будем искать решение уравнения (3.3) в виде

$$
\Phi(\bar{z})=-\frac{1}{2 \pi i} \oint_{\gamma} G(\bar{u}, \bar{z}) p(\bar{u}) d \bar{u}, \quad p \in J,
$$

где $G$ задается формулой (3.7). Под действием отображения (3.8) операторы $\stackrel{0}{S_{1}}, \stackrel{0}{S_{2}}$, $\stackrel{0}{S}_{3}$ преобразуются в операторы $\widehat{S}_{1}, \widehat{S}_{2}, \widehat{S}_{3}$, действующие из $J$ в $J$, а уравнение $(3.3)-$ в уравнение

$$
\left(-\widehat{S}_{2}^{2}+\left[\frac{8 a x_{0}}{1+x_{0}^{2}}+\hbar b_{1}^{(1)}\right] \widehat{S}_{1}+\left[4 a \frac{1-x_{0}^{2}}{1+x_{0}^{2}}+\hbar b_{3}^{(1)}\right] \widehat{S}_{3}-4 a^{2}-\hbar \xi_{k, \ell}^{(1)}\right) p=0 .
$$

Справедлива следующая лемма [19]. 
Лемма 1. Операторы $\widehat{S}_{1}, \widehat{S}_{2}, \widehat{S}_{3}$ имеют вид

$$
\begin{aligned}
& \widehat{S}_{1}=\frac{\hbar}{2}\left(\bar{u} \ell+\left(1-\bar{u}^{2}\right) \frac{d}{d \bar{u}}-\frac{\bar{u}^{\ell}}{\ell !} \frac{d^{\ell+1}}{d \bar{u}^{\ell+1}}\right), \\
& \widehat{S}_{2}=\frac{i \hbar}{2}\left(\bar{u} \ell-\left(1+\bar{u}^{2}\right) \frac{d}{d \bar{u}}+\frac{\bar{u}^{\ell}}{\ell !} \frac{d^{\ell+1}}{d \bar{u}^{\ell+1}}\right), \\
& \widehat{S}_{3}=\hbar\left(\frac{\ell}{2}-\bar{u} \frac{d}{d \bar{u}}\right) .
\end{aligned}
$$

Пусть $\Phi^{\mathrm{WKB}}$ - ВКБ-приближение для решения уравнения (3.3) [27]. Тогда с помощью рассуждений, аналогичных приведенным в работе [19], можно доказать, что если контур $\gamma$ расположен достаточно близко к нулю, то при подстановке $\Phi^{\mathrm{WKB}}$ в уравнение (3.9) дополнительные слагаемые

$$
-\frac{\hbar}{2} \frac{\bar{u}^{\ell}}{\ell !} \frac{d^{\ell+1}}{d \bar{u}^{\ell+1}}, \quad \frac{i \hbar}{2} \frac{\bar{u}^{\ell}}{\ell !} \frac{d^{\ell+1}}{d \bar{u}^{\ell+1}},
$$

появившиеся в результате замены $\stackrel{0}{S_{1}}, \stackrel{0}{S_{2}}, \stackrel{0}{S}_{3}$ на $\widehat{S}_{1}, \widehat{S}_{2}, \widehat{S}_{3}$, при $\ell \rightarrow \infty$ вносят в невязку экспоненциально малый вклад $O\left(\ell^{-\infty} \Phi^{\mathrm{WKB}}\right)$ по сравнению с невязкой $O\left(\ell^{-2} \Phi^{\mathrm{WKB}}\right)$ в случае уравнения (3.3). При этом используются неравенства Коши [28]. Следовательно, вместо асимптотического решения уравнения $(3.9)(\ell+2)$-го порядка в правую часть равенства (3.8) можно подставить асимптотическое решение уравнения второго порядка (3.3).

\section{4. АСИМПТОТИЧЕСКОЕ РЕШЕНИЕ МНОГОТОЧЕЧНОЙ СПЕКТРАЛЬНОЙ ЗАДАЧИ. ВЫЧИСЛЕНИЕ ПОПРАВКИ В СПЕКТРАЛЬНОЙ СЕРИИ}

При построении асимптотики существенную роль играет поведение решений уравнения (2.10) вблизи особых точек. В окрестностях иррегулярных точек $\bar{z}_{1}=i$ и $\bar{z}_{2}=-i$ одно решение представимо в виде суммы степенного ряда, а второе линейно независимое решение задается рядом Лорана. Для регулярной точки $\bar{z}_{3}=\infty$ характеристические показатели равны $-\ell$ и $-\ell+1$, и, следовательно, оба линейно независимых решения уравнения (2.10) растут на бесконечности не быстрее чем $\bar{z}^{\ell}$. Поэтому условие принадлежности решения пространству $\mathcal{P}_{\ell}$ не фиксирует поведение решения на бесконечности.

При $\xi=\xi_{k, \ell}$ точными решениями спектральной задачи $(2.10),(2.11)$ являются многочлены. Наряду с такой задачей рассмотрим многоточечную спектральную задачу. Она состоит в нахождении чисел $\xi_{k, \ell}$ (собственных значений), при которых у уравнения (2.10) существуют ненулевые антиголоморфные решения, которые разлагаются в степенные ряды вблизи особых точек $\bar{z}_{1}=i$ и $\bar{z}_{2}=-i$.

Если число $\xi_{k, \ell}$ и функция $p(\bar{z})$ являются асимптотическим решением такой многоточечной спектральной задачи, то при подстановке $p(\bar{u})$ в правую часть формулы (3.8) получаем многочлен $\Phi(\bar{z})$ - асимптотическое решение уравнения (2.10) из пространства $\mathcal{P}_{\ell}$. Условие нормировки $(2.11)$ для функции $\Phi(\bar{z})$ позволяет определить содержащийся в $p(\bar{u})$ произвольный множитель. Таким образом, число $\xi_{k, \ell}$ 
и многочлен $\Phi(\bar{z})$ являются асимптотическим решением исходной спектральной задачи (2.10), (2.11).

Перейдем к нахождению асимптотических решений уравнения (3.3). Выполним подстановку $\Phi(\bar{z})=E(\bar{z}) Y(\bar{z})$ (см. работу [27]), где

$$
E(\bar{z})=R^{a / \hbar-1 / 2}(\bar{z}) \exp \left(-\frac{4 a\left(1-x_{0}^{2}+2 x_{0} \bar{z}\right)}{\hbar R\left(x_{0}\right) R(\bar{z})}-\frac{b_{3}^{(1)}+b_{1}^{(1)} \bar{z}}{R(\bar{z})}\right) .
$$

Тогда уравнение (3.3) преобразуется к виду

$$
\hbar^{2} \frac{d^{2} Y}{d \bar{z}^{2}}-\left(Q_{0}(\bar{z})+\hbar Q_{1}(\bar{z})+O\left(\hbar^{2}\right)+O\left(\frac{\hbar^{2}}{R^{4}(\bar{z})}\right)\right) Y=0 .
$$

Здесь

$$
\begin{aligned}
Q_{0}(\bar{z})= & 32 a^{2}\left(\bar{z}-x_{0}\right)^{2} \Lambda(\bar{z}) R^{-4}(\bar{z}) R^{-2}\left(x_{0}\right), \\
Q_{1}(\bar{z})= & 4 a\left\{\left[2-2 x_{0}^{2}+4 x_{0} b_{1}^{(1)}+R\left(x_{0}\right) b_{3}^{(1)}+R\left(x_{0}\right) \frac{\xi_{k, \ell}^{(1)}}{a}\right] \bar{z}^{4}+\right. \\
& +\left[-8 x_{0}+2\left(1-3 x_{0}^{2}\right) b_{1}^{(1)}+8 x_{0} b_{3}^{(1)}\right] \bar{z}^{3}+ \\
& +\left[-8 x_{0} b_{1}^{(1)}+8\left(1-x_{0}^{2}\right) b_{3}^{(1)}+2 R\left(x_{0}\right) \frac{\xi_{k, \ell}^{(1)}}{a}\right] \bar{z}^{2}+ \\
& +\left[-8 x_{0}+2\left(x_{0}^{2}-3\right) b_{1}^{(1)}-8 x_{0} b_{3}^{(1)}\right] \bar{z}- \\
& \left.-2+2 x_{0}^{2}+4 x_{0} b_{1}^{(1)}-R\left(x_{0}\right) b_{3}^{(1)}+R\left(x_{0}\right) \frac{\xi_{k, \ell}^{(1)}}{a}\right\} R^{-4}(\bar{z}) R^{-1}\left(x_{0}\right),
\end{aligned}
$$

где

$$
\Lambda(\bar{z})=\left(3 x_{0}^{2}+1\right) \bar{z}^{2}+4 x_{0} \bar{z}+x_{0}^{2}+3,
$$

а многочлен $R(\bar{z})$ задан формулой (3.4).

Приравнивая $Q_{0}(\bar{z})$ к нулю, находим, что уравнение (4.2) имеет точку поворота $\bar{z}=x_{0}$ кратности 2 , а также простые точки поворота

$$
\bar{z}_{ \pm}=\frac{-2 x_{0} \pm i \sqrt{3}\left(1+x_{0}^{2}\right)}{1+3 x_{0}^{2}} .
$$

Точки $\bar{z}_{ \pm}$лежат на окружностях радиуса $1 / \sqrt{3}$ с центрами в точках $\pm 2 i / \sqrt{3}$. При движении $x_{0}$ по вещественной оси от $-\infty$ до $+\infty$ точки $\bar{z}_{ \pm}$совершают полные обороты по указанным окружностям (см. рис 1).

Построим ВКБ-приближения для решений уравнения (4.2). Они справедливы вне малых окрестностей точек поворота и имеют вид

$$
Y_{ \pm}^{\mathrm{WKB}}=\frac{\tilde{c}_{ \pm}}{\sqrt[4]{Q_{0}(\bar{z})}} \exp \left( \pm \frac{1}{\hbar} \int \sqrt{Q_{0}(\bar{z})} d \bar{z} \pm \int \frac{Q_{1}(\bar{z})}{2 \sqrt{Q_{0}(\bar{z})}} d \bar{z}\right)
$$

Здесь $\tilde{c}_{ \pm}-$константы, в степенных функциях берутся главные значения. Разрез между точками поворота $\bar{z}_{-}$и $\bar{z}_{+}$проведен либо вдоль дуги окружности $\bar{z}_{-} \bar{z}_{+}$, проходящей через точки $\bar{z}_{-}, \bar{z}_{+},-1 / x_{0}$, если $x_{0} \neq 0$, либо вдоль лежащих на мнимой 


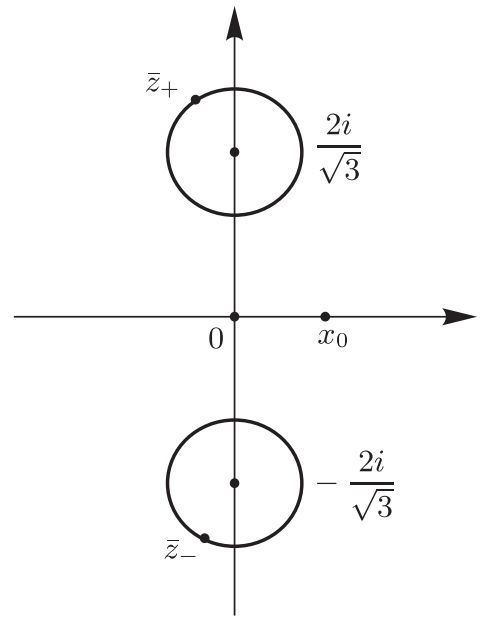

Рис. 1

оси лучей $(\infty,-i \sqrt{3}]$ и $[i \sqrt{3}, \infty)$, если $x_{0}=0$. Отметим, что эти кривые являются линиями Стокса [27]. На рис. $2 \mathrm{a}\left(\right.$ при $\left.x_{0} \neq 0\right)$ и рис. 26 (при $\left.x_{0}=0\right)$, помимо особых точек и точек поворота, изображены линии Стокса. Интегралы в (4.4) выражаются через элементарные функции, однако имеют весьма громоздкий вид.

Вблизи точек поворота $\bar{z}_{+}, \bar{z}_{-}$асимптотические решения уравнения (4.2) выражаются через функции Эйри. А именно, вблизи точек $\bar{z}_{ \pm}$

$$
Y(\bar{z}) \sim \alpha_{1, \pm} \operatorname{Ai}\left(\frac{ \pm \tau_{ \pm}^{2 / 3}\left(i \bar{z}-i \bar{z}_{ \pm}\right)}{\hbar^{2 / 3}}\right)+\alpha_{2, \pm} \operatorname{Bi}\left( \pm \frac{\tau_{ \pm}^{2 / 3}\left(i \bar{z}-i \bar{z}_{ \pm}\right)}{\hbar^{2 / 3}}\right)
$$

Здесь $\alpha_{1, \pm}, \alpha_{2, \pm}-$ константы, $\tau_{ \pm}=2 a 3^{3 / 4}\left(1 \mp i \sqrt{3} x_{0}\right)^{3} R^{-3 / 2}\left(x_{0}\right)$. Вблизи точки поворота $\bar{z}=x_{0}$ асимптотическое решение представимо в виде линейной комбинации функций параболического цилиндра:

$$
Y(\bar{z}) \sim \alpha_{1} D_{\nu}(\bar{u})+\alpha_{2} D_{-\nu-1}(i \bar{u})
$$

где $\alpha_{1}, \alpha_{2}-$ константы и

$$
\begin{gathered}
\bar{u}=\frac{\bar{z}-x_{0}}{\sqrt{\hbar} \beta}, \quad \beta=\frac{\sqrt[4]{2}\left(1+x_{0}^{2}\right)}{4 \sqrt[4]{3} \sqrt{a}}, \\
\nu=\frac{1}{2 \sqrt{6}}\left(-\frac{\xi_{k, \ell}^{(1)}}{a}+2+\frac{1-x_{0}^{2}}{1+x_{0}^{2}} b_{3}^{(1)}+\frac{2 x_{0}}{1+x_{0}^{2}} b_{1}^{(1)}\right)-\frac{1}{2} .
\end{gathered}
$$

Анализ ВКБ-приближений показывает, что функция $\Phi_{-}^{\mathrm{WKB}}=E(\bar{z}) Y_{-}^{\mathrm{WKB}}$ разлагается в степенной ряд вблизи особых точек $\bar{z}_{1}=i$ и $\bar{z}_{2}=-i$. Тем самым определен вид ВКБ-приближения в областях I-VI (см. рис. 2). Произведем согласование асимптотических разложений. Функция $Y_{-}^{\mathrm{WKB}}$ экспоненциально убывает при стремлении $\bar{z}$ к $\bar{z}_{+}$в пределах области I. C другой стороны, в указанной области 


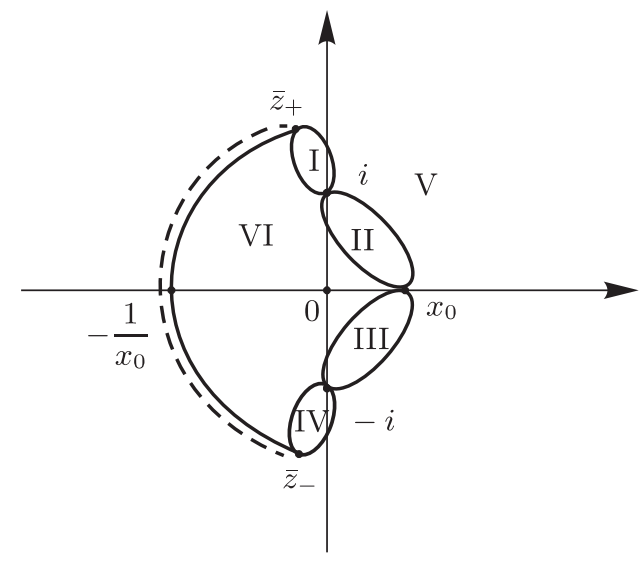

a

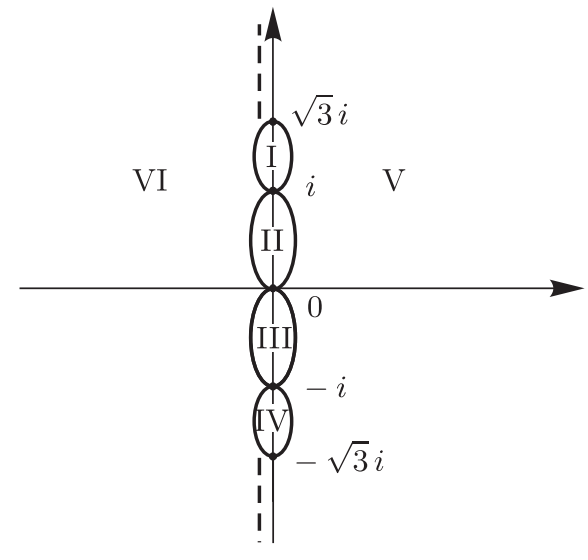

б

Рис. 2

асимптотическое разложение для функций

$$
\operatorname{Ai}\left(\tau_{+}^{2 / 3} \frac{i \bar{z}-i \bar{z}_{+}}{\hbar^{2 / 3}}\right) \text { и } \operatorname{Bi}\left(\tau_{+}^{2 / 3} \frac{i \bar{z}-i \bar{z}_{+}}{\hbar^{2 / 3}}\right)
$$

при $\left|\bar{z}-\bar{z}_{+}\right| \gg \hbar^{2 / 3}$ экспоненциально убывает и возрастает соответственно [29]. Поэтому константа $\alpha_{2,+}$ в формуле (4.5) равна нулю. Аналогично можно показать, что $\alpha_{2,-}=0$.

Асимптотические решения, построенные вблизи точек $\bar{z}_{+}, \bar{z}_{-}$, в областях V и VI также согласуются с $Y_{-}^{\mathrm{WKB}}(\bar{z})$. Таким образом, остается согласовать решения вблизи точки $\bar{z}=x_{0}$. При приближении к точке $\bar{z}=x_{0}$ в пределах области V ВКБ-приближение $Y_{-}^{\mathrm{WKB}}(\bar{z})$ экспоненциально убывает. Воспользуемся асимптотическими разложениями для функций параболического цилиндра [26]. При $|z| \rightarrow \infty$ имеем

$$
\begin{array}{rlr}
D_{\nu}(z)= & z^{\nu} e^{-z^{2} / 4}\left(1+O\left(z^{-2}\right)\right), & -\frac{3 \pi}{4}<\arg z<\frac{3 \pi}{4}, \\
D_{\nu}(z)= & z^{\nu} e^{-z^{2} / 4}\left(1+O\left(z^{-2}\right)\right)- & \pi \\
& -\frac{\sqrt{2 \pi}}{\Gamma(-\nu)} e^{\nu \pi i} z^{-\nu-1} e^{z^{2} / 4}\left(1+O\left(z^{-2}\right)\right), & \frac{\pi}{4}<\arg z<\frac{5 \pi}{4},
\end{array}
$$

где $\Gamma(\nu)$ - гамма-функция. Следовательно, при $-\pi / 4<\arg \bar{u}<\pi / 4$ и $|\bar{u}| \rightarrow \infty$ в формуле (4.6) функция $D_{-\nu-1}(i \bar{u})$ экспоненциально возрастает, а функция $D_{\nu}(\bar{u})$ экспоненциально убывает. Поэтому $\alpha_{2}=0$.

Наконец, при приближении к точке $\bar{z}=x_{0}$ в пределах области VI ВКБ-приближение $Y_{-}^{\mathrm{WKB}}$ также экспоненциально убывает. В силу формулы (4.8) требование экспоненциального убывания функции $D_{\nu}(\bar{u})$ при $3 \pi / 4<\arg \bar{u}<5 \pi / 4$ и $|\bar{u}| \rightarrow \infty$ приводит к условию $1 / \Gamma(-\nu)=0$. Как известно [29], гамма-функция $\Gamma(-\nu)$ имеет 
полюсы лишь при $\nu=k, k=0,1,2, \ldots$. Так как $\nu$ и $\xi_{k, \ell}^{(1)}$ связаны равенством (4.7), поправка в спектральной серии найдена. Таким образом, доказана

Лемма 2. Числа $\xi_{k, \ell}^{(1)}$ в формуле (3.2) имеют вид

$$
\xi_{k, \ell}^{(1)}=a\left(2+\frac{1-x_{0}^{2}}{1+x_{0}^{2}} b_{3}^{(1)}+\frac{2 x_{0}}{1+x_{0}^{2}} b_{1}^{(1)}-2 \sqrt{6}\left(k+\frac{1}{2}\right)\right), \quad k=0,1,2, \ldots .
$$

При $\nu=k$ функции параболического цилиндра выражаются через полиномы Эрмита. Поэтому для асимптотики вблизи точки $\bar{z}=x_{0}$ имеем

$$
\begin{aligned}
Y(\bar{z}) \sim & \frac{\alpha_{1}}{2^{k / 2}} H_{k}\left(\frac{\bar{u}}{\sqrt{2}}\right) e^{-\bar{u}^{2} / 4}+ \\
& +\frac{\sqrt{\hbar} \alpha_{1}}{\sqrt{a} 2^{(k+3) / 2}} \sqrt[4]{\frac{2}{3}}\left\{\left[x_{0}\left(\frac{\bar{u}}{\sqrt{2}}\right)^{3}+\left(x_{0}-B\left(x_{0}\right)\right) \frac{\bar{u}}{\sqrt{2}}\right] H_{k}\left(\frac{\bar{u}}{\sqrt{2}}\right)+\right. \\
& \left.+\left[-x_{0}\left(\frac{\bar{u}}{\sqrt{2}}\right)^{2}+B\left(x_{0}\right)\right] H_{k}^{\prime}\left(\frac{\bar{u}}{\sqrt{2}}\right)\right\} e^{-\bar{u}^{2} / 4} .
\end{aligned}
$$

Здесь

$$
B\left(x_{0}\right)=\sqrt{\frac{3}{2}}\left(\frac{2 x_{0}}{1+x_{0}^{2}} b_{3}^{(1)}-\frac{1-x_{0}^{2}}{1+x_{0}^{2}} b_{1}^{(1)}\right) .
$$

Формула (4.10), помимо главного члена, содержит также поправку. Наконец, при выполнении равенства (4.9) формула для ВКБ-приближения принимает вид

$$
\begin{aligned}
& \Phi_{-}^{\mathrm{WKB}}(\bar{z})=\frac{c_{-}\left(\bar{z}-x_{0}\right)^{k}}{\sqrt[4]{\Lambda(\bar{z})}} \frac{\left(\sqrt{\Lambda(\bar{z})}+\sqrt{2}\left(x_{0} \bar{z}+1\right)\right)^{\ell+1}}{\left(\sqrt{\Lambda(\bar{z})}+\sqrt{3}\left(x_{0} \bar{z}+1\right)\right)^{k+1 / 2}} \times \\
& \quad \times \exp \left(\frac{\sqrt{2} \ell\left[x_{0}^{2}\left(1+3 x_{0}^{2}\right) \bar{z}^{2}+2 x_{0}\left(1+5 x_{0}^{2}\right) \bar{z}+1+5 x_{0}^{2}-2 x_{0}^{4}\right]}{\left(1+x_{0}^{2}\right)\left[\left(x_{0} \bar{z}+1\right) \sqrt{\Lambda(\bar{z})}+\sqrt{2}\left(2 x_{0} \bar{z}+1-x_{0}^{2}\right)\right]}+\frac{\Lambda_{1}(\bar{z})}{\Lambda_{2}(\bar{z})}\right),
\end{aligned}
$$

где $k=0,1,2, \ldots$, величина $c_{-}-$константа, $x_{0} \geqslant 0$, многочлен $\Lambda(\bar{z})$ задан формулой $(4.3)$ и

$$
\begin{aligned}
\Lambda_{1}(\bar{z})= & \left(1+3 x_{0}^{2}\right)\left(b_{1}^{(1)}-x_{0} b_{3}^{(1)}\right)^{2} \bar{z}^{2}+2\left(1+x_{0}^{2}\right)\left(b_{1}^{(1)}-x_{0} b_{3}^{(1)}\right)\left(b_{3}^{(1)}+3 x_{0} b_{1}^{(1)}\right) \bar{z}+ \\
& +\left(3+x_{0}^{2}\right)\left(b_{3}^{(1)}+x_{0} b_{1}^{(1)}\right)^{2}-2\left(1+x_{0}^{2}\right)^{2}\left(b_{3}^{(1)}\right)^{2}, \\
\Lambda_{2}(\bar{z})= & \sqrt{2}\left(1+x_{0}^{2}\right)\left[\left(\left(b_{1}^{(1)}-x_{0} b_{3}^{(1)}\right) \bar{z}+b_{3}^{(1)}+x_{0} b_{1}^{(1)}\right) \sqrt{\Lambda(\bar{z})}+\sqrt{2}\left(1+x_{0}^{2}\right)\left(b_{1}^{(1)} \bar{z}+b_{3}^{(1)}\right)\right] .
\end{aligned}
$$

Формула для ВКБ-приближения в случае $x_{0}<0$ получается в результате замены в правой части равенства (4.11) $\bar{z}, x_{0}, b_{1}^{(1)}$ на $-\bar{z},-x_{0},-b_{1}^{(1)}$.

Таким образом, асимптотическое решение многоточечной спектральной задачи построено (константы $b_{1}^{(1)}, b_{3}^{(1)}$ будут вычислены ниже). Числа $\xi_{k, \ell}$ задаются формулами $(3.2),(4.9)$, а антиголоморфная функция $p(\bar{z})$ получается в результате согласования асимптотик. А именно, $p(\bar{z})=\Phi_{-}^{\mathrm{WKB}}(\bar{z})$ является асимптотическим решением уравнения (3.3) на всей комплексной плоскости, за исключением малых окрестностей точек поворота $\bar{z}_{+}, \bar{z}_{-}, x_{0}$, а также дуги $\bar{z}_{-} \bar{z}_{+}($либо лучей $(\infty,-i \sqrt{3}],[i \sqrt{3}, \infty)$ при $\left.x_{0}=0\right)$. Вблизи точек поворота $\bar{z}_{+}, \bar{z}_{-}$и $x_{0}$ мы имеем $p(\bar{z})=E(\bar{z}) Y(\bar{z})$, где $Y(\bar{z})$ задается формулами (4.5) (при $\alpha_{2, \pm}=0$ ) и (4.10) соответственно. Асимптотики согласуются между собой на расстояниях порядка $\hbar^{1 / 2}$ от точек $\bar{z}_{ \pm}$и расстояниях порядка $\hbar^{3 / 8}$ от точки $x_{0}$. 


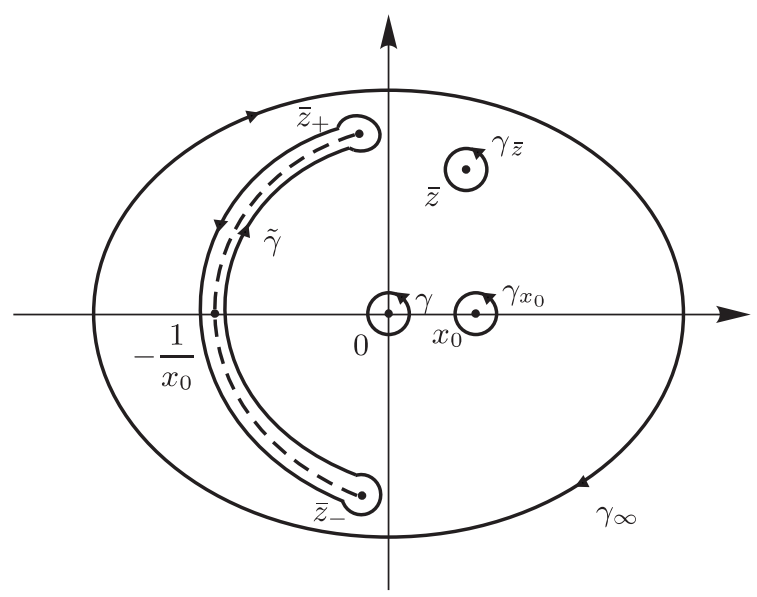

Рис. 3

\section{5. АСИМПТОТИКА МНОГОЧЛЕНОВ $\Phi(\bar{Z})$}

Подставим асимптотическое решение $p(\bar{z})$ многоточечной спектральной задачи в правую часть формулы (3.8) и вычислим асимптотику возникающего интеграла. Пусть для определенности $x_{0} \neq 0$. Подынтегральная функция в равенстве (3.8) не имеет точек перевала. Поэтому воспользуемся интегральной теоремой Коши, согласно которой сумма интегралов по контурам $\gamma, \gamma_{\bar{z}}, \tilde{\gamma}, \gamma_{x_{0}}$ и $\gamma_{\infty}$ равна нулю (см. рис. 3).

Интегралы по контурам $\gamma_{x_{0}}$ и $\gamma_{\infty}$ равны нулю в силу антиголоморфности подынтегральной функции и теоремы о вычетах, а интеграл по $\gamma_{\bar{z}}$ вычисляется с помощью интегральной формулы Коши, таким образом, при значениях $\bar{z}$, не лежащих на дуге $\bar{z}_{-} \bar{z}_{+}$, и таких, что $\left|\bar{z}-x_{0}\right| \gtrsim \hbar^{3 / 8},\left|\bar{z}-\bar{z}_{ \pm}\right| \gtrsim \hbar^{1 / 2}$, многочлен $\Phi(\bar{z})$ можно представить в виде

$$
\Phi(\bar{z})=\Phi_{-}^{\mathrm{WKB}}(\bar{z})+N(\bar{z})
$$

где

$$
N(\bar{z})=-\frac{\bar{z}^{\ell+1}}{2 \pi i} \oint_{\gamma_{+,-}} \frac{\Phi_{-}^{\mathrm{WKB}}(\bar{u}) d \bar{u}}{\bar{u}^{\ell+1}(\bar{u}-\bar{z})} .
$$

Здесь замкнутый контур $\gamma_{+,-}$является дугой окружности $\bar{z}_{-} \bar{z}_{+}$и проходится дважды по берегам разреза, соединяющего точки $\bar{z}_{-}, \bar{z}_{+}$. Этот контур ориентирован против часовой стрелки. Формула, аналогичная (5.1), справедлива и вблизи точки $\bar{z}=x_{0}$, но в этом случае вместо $\Phi_{-}^{\mathrm{WKB}}(\bar{z})$ в ней фигурирует $E(\bar{z}) Y(\bar{z})$, где $Y(\bar{z})-$ разложение (4.10).

Оценим входящий в формулу (5.2) интеграл. Имеем

$$
|N(\bar{z})| \leqslant \frac{|\bar{z}|^{\ell+1}}{2 \pi} \oint_{\gamma_{+,-}} \frac{\left|\Phi_{-}^{\mathrm{WKB}}(\bar{u})\right||d \bar{u}|}{|\bar{u}|^{\ell+1}|\bar{u}-\bar{z}|} .
$$


Так как $\bar{z}_{-}^{\smile} \bar{z}_{+}-$линия Стокса, на ней в силу соотношения (4.1) справедливо равенство

$$
\frac{\left|\Phi_{-}^{\mathrm{WKB}}(\bar{u})\right|}{|\bar{u}|^{\ell+1}}=\frac{\left(1+x_{0}^{2}\right)^{\ell / 2} e^{\ell \Psi(\bar{u}) / 2} \vartheta(\bar{u})}{\sqrt[4]{|\Lambda(\bar{u})|}}
$$

где

$$
\Psi(\bar{u})=\ln \left|1+\bar{u}^{2}\right|-\frac{4\left(1-x_{0}^{2}+2 x_{0} \bar{u}\right)}{\left(1+x_{0}^{2}\right)\left(1+\bar{u}^{2}\right)}-2 \ln |\bar{u}|,
$$

а $\vartheta(\bar{u})$ - непрерывная ограниченная функция.

При $0<\left|x_{0}\right|<\sqrt{2 e^{2}-3}$

$$
\max _{\bar{u} \in \bar{z}_{-} \bar{z}_{+}} \Psi(\bar{u})=\ln \left(\frac{2\left(1+x_{0}^{2}\right)}{3+x_{0}^{2}}\right)+\frac{2\left(1+3 x_{0}^{2}\right)}{1+x_{0}^{2}}
$$

и достигается в точках поворота $\bar{z}_{ \pm}$, а при $\left|x_{0}\right| \geqslant \sqrt{2 e^{2}-3}$

$$
\max _{\bar{u} \in \bar{z}_{-} \bar{z}_{+}} \Psi(\bar{u})=\ln \left(1+x_{0}^{2}\right)+\frac{4 x_{0}^{2}}{1+x_{0}^{2}}
$$

и достигается в точке $-1 / x_{0}$, если $\left|x_{0}\right|>\sqrt{2 e^{2}-3}$, и в точках $\bar{z}_{-}, \bar{z}_{+},-1 / x_{0}$, если $\left|x_{0}\right|=\sqrt{2 e^{2}-3}$. Наибольший вклад в асимптотику интеграла (5.3) вносят малые окрестности этих точек. Применяя метод Лапласа, а также используя интеграл [30]

$$
\int_{0}^{\infty} x^{-1 / 4} e^{-\ell x} d x=\ell^{-3 / 4} \Gamma\left(\frac{3}{4}\right),
$$

получаем следующее утверждение.

ЛЕмма 3. При $\ell \rightarrow \infty, \bar{z} \notin \bar{z}_{-} \bar{z}_{+}$справедливы следующие оценки: если $0<\left|x_{0}\right|<\sqrt{2 e^{2}-3}$, mo

$$
\begin{aligned}
|N(\bar{z})| \leqslant & \frac{T\left|c_{-}\right|}{\ell^{3 / 4}}\left(1+x_{0}^{2}\right)^{\ell}\left(\frac{2}{3+x_{0}^{2}}\right)^{\ell / 2} \exp \left(\frac{\ell\left(1+3 x_{0}^{2}\right)}{1+x_{0}^{2}}\right) \times \\
& \times|\bar{z}|^{\ell+1}\left(\frac{1}{\left|\bar{z}-\bar{z}_{+}\right|}+\frac{1}{\left|\bar{z}-\bar{z}_{-}\right|}\right) ;
\end{aligned}
$$

ecлu $\left|x_{0}\right|=\sqrt{2 e^{2}-3}$, mo

$$
\begin{aligned}
|N(\bar{z})| \leqslant & \frac{T\left|c_{-}\right|}{\ell^{3 / 4}}\left(1+x_{0}^{2}\right)^{\ell} \exp \left(\frac{\ell 2 x_{0}^{2}}{1+x_{0}^{2}}\right) \times \\
& \times|\bar{z}|^{\ell+1}\left(\frac{1}{\left|\bar{z}-\bar{z}_{+}\right|}+\frac{1}{\left|\bar{z}-\bar{z}_{-}\right|}+\frac{\ell^{1 / 4}}{\left|\bar{z}+1 / x_{0}\right|}\right)
\end{aligned}
$$

ecлu $\left|x_{0}\right|>\sqrt{2 e^{2}-3}$, mo

$$
|N(\bar{z})| \leqslant \frac{T\left|c_{-}\right|}{\sqrt{\ell}}\left(1+x_{0}^{2}\right)^{\ell} \exp \left(\frac{\ell 2 x_{0}^{2}}{1+x_{0}^{2}}\right) \frac{|\bar{z}|^{\ell+1}}{\left|\bar{z}+1 / x_{0}\right|} .
$$

Здесъ T-константа. 
Отметим, что и при $x_{0}=0$ имеет место формула, аналогичная (5.1). Надо лишь контур $\gamma_{+,-}$в формуле (5.2) заменить на лучи $(\infty,-i \sqrt{3}],[i \sqrt{3}, \infty)$, которые проходятся дважды по берегам разреза против часовой стрелки. Снова наибольший вклад в интеграл (5.3) дадут малые окрестности точек поворота $\pm i \sqrt{3}$. В результате получается следующая асимптотика для $N(\bar{z})$ :

$$
N(\bar{z}) \sim \frac{c_{-} e^{\ell}}{\ell^{3 / 4}}\left(\frac{2}{3}\right)^{\ell / 2}\left(\frac{T_{1}}{\bar{z}-i \sqrt{3}}+\frac{T_{2}}{\bar{z}+i \sqrt{3}}\right) .
$$

Здесь $T_{1}$ и $T_{2}$ - некоторые константы.

Рассмотрим окрестность точки $\bar{z}=x_{0}$, которая дает основной вклад в асимптотику средних, и найдем, при каких $x_{0}$ значения функции $N(\bar{z})$ в этой окрестности будут экспоненциально малыми по сравнению с $p(\bar{z})=E(\bar{z}) Y(\bar{z})$, где $Y(\bar{z})$ - разложение (4.10). Так как это разложение согласуется с ВКБ-приближением, из соотношения (4.11) вытекает, что порядок значений $p(\bar{z})$ вблизи точки $x_{0}$ определяется множителем

$$
\left(1+x_{0}^{2}\right)^{\ell}(\sqrt{3}+\sqrt{2})^{\ell} \exp \left(\frac{\sqrt{2} \ell\left(3 x_{0}^{4}+6 x_{0}^{2}+1\right)}{\left(1+x_{0}^{2}\right)\left(\sqrt{3}\left(1+x_{0}^{2}\right)+\sqrt{2}\right)}\right) .
$$

Сравнивая это выражение с правыми частями формул (5.4)-(5.7), находим, что для экспоненциальной малости величины $N(\bar{z})$ по сравнению с $p(\bar{z})$ при

$$
0 \leqslant\left|x_{0}\right|<\sqrt{2 e^{2}-3}
$$

должно выполняться неравенство

$$
\frac{\sqrt{2}\left|x_{0}\right|}{\sqrt{3+x_{0}^{2}}} \exp \left(\frac{1+3 x_{0}^{2}}{1+x_{0}^{2}}\right)<(\sqrt{3}+\sqrt{2}) \exp \left(\frac{\sqrt{2}\left(3 x_{0}^{4}+6 x_{0}^{2}+1\right)}{\left(1+x_{0}^{2}\right)\left(\sqrt{3}\left(1+x_{0}^{2}\right)+\sqrt{2}\right)}\right)
$$

а при $\left|x_{0}\right| \geqslant \sqrt{2 e^{2}-3}-$ неравенство

$$
\left|x_{0}\right| \exp \left(\frac{2 x_{0}^{2}}{1+x_{0}^{2}}\right)<(\sqrt{3}+\sqrt{2}) \exp \left(\frac{\sqrt{2}\left(3 x_{0}^{4}+6 x_{0}^{2}+1\right)}{\left(1+x_{0}^{2}\right)\left(\sqrt{3}\left(1+x_{0}^{2}\right)+\sqrt{2}\right)}\right) .
$$

Неравенство (5.9) имеет место при всех $x_{0}$, удовлетворяющих неравенству (5.8), а неравенство (5.10) выполняется лишь при

$$
\left|x_{0}\right|<x_{*}
$$

где $x_{*}-$ корень уравнения

$$
x \exp \left(\frac{2 x^{2}}{1+x^{2}}\right)=(\sqrt{3}+\sqrt{2}) \exp \left(\frac{\sqrt{2}\left(3 x^{4}+6 x^{2}+1\right)}{\left(1+x^{2}\right)\left(\sqrt{3}\left(1+x^{2}\right)+\sqrt{2}\right)}\right),
$$

приближенное значение которого $x_{*} \approx 4.94$. Это связано с тем, что для $N(\bar{z})$ выше была использована довольно грубая оценка сверху (5.6). 
Далее мы ограничимся рассмотрением значений $x_{0}$, которые удовлетворяют условию (5.11). Для нахождения асимптотических собственных функций при $\left|x_{0}\right| \geqslant x_{*}$ можно воспользоваться связью между изменением величины $x_{0}$ и сдвигом на полярный угол, который допускают собственные функции задачи (1.1). Сдвиг на угол $\varphi_{0}$ порождает в пространстве $\mathcal{P}_{\ell}$ оператор $M_{\varphi_{0}}$, действующий согласно формуле

$$
M_{\varphi_{0}} g(\bar{z})=\left(\cos \varphi_{0}-\bar{z} \sin \varphi_{0}\right)^{\ell} g\left(\frac{\bar{z} \cos \varphi_{0}+\sin \varphi_{0}}{\cos \varphi_{0}-\bar{z} \sin \varphi_{0}}\right)
$$

\section{6. ФОРМУЛЫ ДЛЯ КВАНТОВЫХ СРЕДНИХ. ИТОГОВАЯ ТЕОРЕМА}

Пусть многочлен $\Phi(\bar{z})$ задан формулой (3.8), где функция $p(\bar{u})$ есть асимптотическое решение многоточечной спектральной задачи. Предположим, что выполнено условие $(5.11)$, и вычислим асимптотику нормы функции $\Phi(\bar{z})$ в пространстве $\mathcal{P}_{\ell}$.

Запишем ВКБ-приближение (4.11) в виде

$$
\Phi_{-}^{\mathrm{WKB}}(\bar{z})=c_{-} t(\bar{z}) e^{\ell s(\bar{z})} .
$$

Если подставить это выражение в формулу (2.8) для скалярного произведения, то получим интеграл

$$
\left\|\Phi_{-}^{\mathrm{WKB}}\right\|_{\mathcal{P}_{\ell}}^{2}=\frac{\ell+1}{2 \pi} \int_{\mathbb{C}} \frac{\left|c_{-}\right|^{2}|t(\bar{z})|^{2} e^{\ell \Omega(\bar{z}, z)}}{\left(1+|z|^{2}\right)^{2}} d \bar{z} d z
$$

где $\Omega(\bar{z}, z)=s(\bar{z})+s(z)-\ln \left(1+|z|^{2}\right)$. Так как функция $\Omega(\bar{z}, z)$ достигает глобального максимума при $\bar{z}=z=x_{0}$, интеграл (6.1) будет приближенно равен интегралу по малой окрестности этой точки.

Далее разложим заданную формулой (4.1) функцию $E(\bar{z})$ вблизи точки $\bar{z}=x_{0}$ и подставим в формулу (2.8) произведение этого разложения на $Y(\bar{z})$, заданную в (4.10). Также учтем, что $N(\bar{z})$ дает экспоненциально малый вклад в норму. В результате получаем, что если входящая в правую часть формулы (4.10) константа $\alpha_{1}$ имеет вид

$$
\alpha_{1}=\sqrt{\pi} 2^{1+k / 2} \sqrt[4]{\frac{3}{2}} \sqrt{1+x_{0}^{2}} \exp \left(\frac{2 \ell+b_{1}^{(1)} x_{0}+b_{3}^{(1)}}{1+x_{0}^{2}}\right) \Sigma_{0}^{-1 / 2}(k)(1+O(\hbar))
$$

где

$$
\Sigma_{j}(k)=\int_{\mathbb{R}^{2}} e^{-(1-\sqrt{2 / 3}) t^{2}-(\sqrt{3 / 2}-1) r^{2}} \sigma_{j}(t, r)\left|H_{k}(t+i r)\right|^{2} d t d r, \quad j=0,1,
$$

и $\sigma_{0}=1$, то условие $\|\Phi(\bar{z})\|_{\mathcal{P}_{\ell}}=1+O(\hbar)$ при $\hbar \rightarrow 0$ будет выполнено. Таким образом, мы нашли входящий в $p$ множитель $\alpha_{1}$. 
Чтобы завершить построение $\Phi(\bar{z})$, остается вычислить значения $b_{1}^{(1)}$ и $b_{3}^{(1)}$. Они задаются квантовыми средними (2.6). Вычисление квантовых средних производится аналогично вычислению нормы. Основной вклад в асимптотику интегралов также вносит малая окрестность точки $\bar{z}=z=x_{0}$ [19]. Справедлива

ЛЕмма 4. При $九 \rightarrow 0$ имеют место равенства

$$
\begin{aligned}
& b_{1}=4\left(\stackrel{0}{S}_{1} \Phi_{k, \ell}, \Phi_{k, \ell}\right) \mathcal{P}_{\ell}=\frac{8 a x_{0}}{1+x_{0}^{2}}+\hbar\left\{\frac{1-x_{0}^{2}}{1+x_{0}^{2}}\left[\frac{1-x_{0}^{2}}{1+x_{0}^{2}} b_{1}^{(1)}-\frac{2 x_{0}}{1+x_{0}^{2}} b_{3}^{(1)}\right]+\right. \\
& \left.+\frac{8 x_{0}}{1+x_{0}^{2}}-\frac{4 x_{0} \Sigma_{1}(k)}{\sqrt{6}\left(1+x_{0}^{2}\right) \Sigma_{0}(k)}\right\}+O\left(\hbar^{2}\right) \text {, } \\
& \left.b_{3}=4\left(\stackrel{0}{S}_{3} \Phi_{k, \ell}, \Phi_{k, \ell}\right)\right)_{\mathcal{P}_{\ell}}=\frac{4 a}{1+x_{0}^{2}}+\hbar\left\{\frac{2 x_{0}}{1+x_{0}^{2}}\left[\frac{2 x_{0}}{1+x_{0}^{2}} b_{3}^{(1)}-\frac{1-x_{0}^{2}}{1+x_{0}^{2}} b_{1}^{(1)}\right]+\right. \\
& \left.+4 \frac{1-x_{0}^{2}}{1+x_{0}^{2}}-\frac{2\left(1-x_{0}^{2}\right) \Sigma_{1}(k)}{\sqrt{6}\left(1+x_{0}^{2}\right) \Sigma_{0}(k)}\right\}+O\left(\hbar^{2}\right) \text {. }
\end{aligned}
$$

Здесъ функция $\Sigma_{1}(k)$ задана формулой (6.3), где $\sigma_{1}=t^{2}+r^{2}$.

В результате сравнения разложений (3.1) с (6.4) получаем соотношение, связывающее $b_{1}^{(1)}$ и $b_{3}^{(1)}$ :

$$
\frac{2 x_{0}}{1+x_{0}^{2}} b_{1}^{(1)}+\frac{1-x_{0}^{2}}{1+x_{0}^{2}} b_{3}^{(1)}=4-\frac{2 \Sigma_{1}(k)}{\sqrt{6} \Sigma_{0}(k)} .
$$

В силу этого равенства коэффициенты $b_{1}^{(1)}, b_{3}^{(1)}$ можно представить в виде

$$
\begin{aligned}
& b_{1}^{(1)}=\frac{2 x_{0}}{1+x_{0}^{2}}\left[4-\frac{2 \Sigma_{1}(k)}{\sqrt{6} \Sigma_{0}(k)}\right]+\frac{8\left(1-x_{0}^{2}\right) x_{1}}{\left(1+x_{0}^{2}\right)^{2}}, \\
& b_{3}^{(1)}=\frac{1-x_{0}^{2}}{1+x_{0}^{2}}\left[4-\frac{2 \Sigma_{1}(k)}{\sqrt{6} \Sigma_{0}(k)}\right]-\frac{16 x_{0} x_{1}}{\left(1+x_{0}^{2}\right)^{2}},
\end{aligned}
$$

где $x_{1}$ - произвольная вещественная константа, которая определяет поправку порядка $\hbar$ к числу $x_{0}$, около которого локализовано решение (т. е. $\bar{z}=x_{0}+\hbar x_{1}$ ). Отметим, что именно через параметры $b_{1}^{(1)}$ и $b_{3}^{(1)}$ функция $\Phi_{-}^{\mathrm{WKB}}$ зависит от величин

$$
\frac{\Sigma_{1}(k)}{\Sigma_{0}(k)}, \quad k=0,1,2, \ldots,
$$

характеризующих решение вблизи точки $x_{0}$.

Наконец, с учетом равенства (6.5) формула (4.9) принимает вид

$$
\xi_{k, \ell}^{(1)}=a\left(6-2 \sqrt{6}\left(k+\frac{1}{2}\right)-\frac{2 \Sigma_{1}(k)}{\sqrt{6} \Sigma_{0}(k)}\right), \quad k=0,1,2, \ldots .
$$

Справедлива

TEOPEMA 1. Пусть

$$
\xi_{k, \ell}=4 a^{2}+\frac{2 a^{2}}{\ell}\left(6-2 \sqrt{6}\left(k+\frac{1}{2}\right)-\frac{2 \Sigma_{1}(k)}{\sqrt{6} \Sigma_{0}(k)}\right)+O\left(\ell^{-2}\right), \quad k=0,1,2, \ldots,
$$


а многочлен $\Phi_{k, \ell}(\bar{z})$ определен формулой $(3.8)$, где $p(\bar{u})$ - решение многоточечной спектральной задачи такое, что $\alpha_{1} u b_{1}^{(1)}, b_{3}^{(1)}$ имеют вид (6.2) u (6.6) cоответственно. Тогда при выполнении условия (5.11) $\xi_{k, \ell} u \Phi_{k, \ell}(\bar{z})$ являются асимптотическим собственным значением и асимптотической собственной функиией задачи (2.10), (2.11) при $\ell \rightarrow \infty$ в пространстве $\mathcal{P}_{\ell}$. Более точно, если $\xi_{k, \ell}$ имеет вид (6.7), то многочлен $\Phi_{k, \ell}(\bar{z})$ удовлетворяет уравнению (2.10) с точностью $O\left(\ell^{-2}\right)$ с оценкой невязки по норме пространства $\mathcal{P}_{\ell}$, a также условию нормировки (2.11) с точностью $O\left(\ell^{-1}\right)$.

В заключение найдем асимптотические собственные значения задачи (1.1), расположенные вблизи верхних границ спектральных кластеров, образующихся вокруг собственных значений невозмущенного оператора.

ЛЕмма 5. При $九 \rightarrow 0$ справедливо равенство

$$
\left(\stackrel{0}{S}_{2}^{2} \Phi_{k, \ell}, \Phi_{k, \ell}\right)_{\mathcal{P}_{\ell}}=2 a \hbar\left[1+\sqrt{6}\left(k+\frac{1}{2}\right)-\frac{\Sigma_{1}(k)}{\sqrt{6} \Sigma_{0}(k)}\right]+O\left(\hbar^{2}\right) .
$$

Подставляя далее соотношения (6.7), (6.8), (2.5) в формулу (2.2), для значений $\ell$ порядка $\hbar^{-1}$ получаем, что при $\hbar \rightarrow 0$

$$
\begin{aligned}
\lambda= & \lambda_{k, \ell}=\ell \hbar+\hbar+\left(w_{0}+2 \ell \hbar w_{1}+9 \ell^{2} \hbar^{2} w_{2}\right) \hbar^{2}+ \\
& +\left[2 w_{1}+2 \ell \hbar w_{2}\left(9-2 \sqrt{6}\left(k+\frac{1}{2}\right)\right)\right] \hbar^{3}+O\left(\hbar^{4}\right), \quad k=0,1,2, \ldots .
\end{aligned}
$$

Полученная серия описывает расщепление спектра оператора типа Хартри и содержит члены вплоть до третьего порядка по $\hbar$ включительно. Соответствующие асимптотические собственные функции задачи (1.1) получаются из многочленов $\Phi_{k, \ell}(\bar{z})$, заданных формулой $(3.8)$, путем применения когерентного преобразования (2.7), а также преобразования (2.3).

Благодарности. Автор благодарен М.В. Карасеву за привлечение внимания к данной задаче, а также за ценные вопросы и замечания. Работа выполнена при поддержке РФФИ (грант № 12-01-00627), Министерства образования и науки РФ (соглашение 14.В37.21.0864) и Программы поддержки ведущих научных школ (грант НШ-2033.2012.1).

\section{Список литературы}

[1] Л. П. Питаевский, УФН, 168:6 (1998), 641-653.

[2] А.С. Давыдов, Солитоны в молекулярных системах, Наукова думка, Киев, 1984.

[3] В. П. Маслов, Комплексный метод ВКБ в нелинейных уравнениях, Наука, М., 1977.

[4] И. В. Сименог, ТМФ, 30:3 (1977), 408-414.

[5] М. В. Карасев, Квантовая редукиия на орбиты алгебр симметрий и задача Эренфеста, Препринт ИТФ-87-157Р, ИТФ АН УССР, Киев, 1987.

[6] С. А. Вакуленко, В.П. Маслов, И. А. Молотков, И. А. Шафаревич, Докл. РАН, 345:6 (1995), 743-745.

[7] М. В. Карасев, А. В. Перескоков, Изв. РАН. Сер. матем., 65:5 (2001), 33-72.

[8] М. В. Карасев, А. В. Перескоков, Изв. РАН. Сер. матем., 65:6 (2001), 57-98.

[9] А.В. Перескоков, ТМФ, 131:3 (2002), 389-406. 
[10] В. В. Белов, Е. И. Смирнова, Матем. заметки, 80:2 (2006), 309-312.

[11] В. В. Белов, Ф. Н. Литвинец, А. Ю. Трифонов, ТМФ, 150:1 (2007), 26-40.

[12] А. Л. Лисок, А. Ю. Трифонов, А. В. Шаповалов, Изв. Томск. политехн. ун-та, 314:2 (2009), 66-71.

[13] В. М. Бабич, В. С. Булдырев, Асимптотические методы в дифракиии коротких волн. Метод эталонных задач, Наука, М., 1972.

[14] М. В. Карасев, В. П. Маслов, УМН, 39:6(240) (1984), 115-173.

[15] M. V. Karasev, "Birkhoff resonances and quantum ray method", Proceedings of International Seminar "Days of Diffraction-2004", St. Petersburg University and Steklov Math. Institute, St. Petersburg, 2004, 114-126.

[16] M. V. Karasev, "Noncommutative algebras, nano-structures, and quantum dynamics generated by resonances, I", Quantum Algebras and Poisson Geometry in Mathematical Physics, American Mathematical Society Translations Series 2, 216, AMS, Providence, RI, 2005, 1-18; Adv. Stud. Contemp. Math., 11:1 (2005), 33-56; Russ. J. Math. Phys., 13:2 (2006), $131-150$.

[17] M. Karasev, "Resonance gyrons and quantum geometry", From Geometry to Quantum Mechanics. In Honor of Hideki Omori, Progress in Mathematics, 252, eds. Y. Maeda, T. Ochiai, P. Michor, A. Yoshioka, Birkäuser, Boston, 2007, 253-275.

[18] А. В. Перескоков, Матем. заметки, 92:4 (2012), 583-596.

[19] А. В. Перескоков, Изв. РАН. Сер. матем., 77:1 (2013), 165-210.

[20] А. В. Перескоков, Тр. ММО, 73:2 (2012), 277-325.

[21] А. В. Перескоков, НМФМ, 8:1 (2013), 65-84.

[22] В. В. Голубев, Лекции по аналитической теории дифференииалъных уравнений, Гостехиздат, М.-Л., 1950.

[23] A. Weinstein, Duke Math. J., 44:4 (1977), 883-892.

[24] J. Schwinger, "On angular momentum", Quantum Theory of Angular Momentum, eds. L. C. Biedenham, H. van Dam, Academic Press, New York, 1965, 229-279.

[25] M. V. Karasev, E. M. Novikova, "Non-Lie permutation relations, coherent states, and quantum embedding", Coherent Transform, Quantization, and Poisson Geometry, American Mathematical Society Translations Series 2, 187, ed. M. V. Karasev, AMS, Providence, RI, 1998, 1-202.

[26] Г. Бейтмен, А. Эрдейи, Высшие трансцендентные функиии, т. 2: Функиии Бесселя, функиии параболического ицлиндра, ортогональные многочлены, Наука, М., 1974.

[27] М. В. Федорюк, Асимптотические методы для линейных обыкновенных дифференциальных уравнений, Наука, М., 1983.

[28] М. А. Лаврентьев, Б. В. Шабат, Методы теории функиий комплексного переменного, Наука, М., 1987.

[29] М. Абрамовиц, И. Стиган (ред.), Справочник по специальным функииям с формулами, графиками и математическими таблицами, Наука, М., 1979.

[30] М.В. Федорюк, Асимптотика: интеграль и ряды, Наука, М., 1987.

Поступила в редакцию 9.06.2013 